\title{
Iterative Method for Solving Nonlinear Inverse Problems of Structural Element Shaping
}

\author{
K. S. Bormotin, S. V. Belykh \\ Komsomolsk-na-Amure State Technical University \\ Russia
}

\begin{abstract}
In the given work the generalized variation formulation quasi-static problems plastic forming details taking into account creep deformations is resulted. For the considered formulation theorems of uniqueness, stability and the convergence of an iterative method proved for inverse problems forming are true. An iterative method is proposed for solving nonlinear inverse problems of shaping structural elements. The method is implemented using a software package based on finite element analysis. Numerical results of problems forming are resulted.
\end{abstract}

Keywords-inverse problems forming; variation inequalities; plasticity; creep; iterative methods; convergence conditions; a method of finite elements

\section{INTRODUCTION}

In connection with introduction of new technological processes, modes, materials at details manufacturing of is difficult-constructive forms with high requirements to dimensional accuracy and an operational resource application of numerical modeling becomes the most favorable. Still recently the establishment of the basic parities of mathematical models demanded accumulation and the analysis of huge files of experimental and natural industrial data that is interfaced to considerable material and time expenses. At the same time the modern mechanical engineering is characterized by more and more increasing frequency of removability of objects of manufacture, and also complication of constructive forms and increase in overall dimensions of details of designs. Thus, the effective decision is application of numerical modeling at reception of estimations operational and technical characteristics on a developed product.

The inverse shaping problem determines external forces and kinematic effects under which a strain process occurring under creep conditions over a given time interval leads to a prescribed residual configuration after elastic unloading [1,2].

By using equations for virtual works under certain constraints, the geometrically nonlinear inverse shaping problems for inelastic plates and shells were shown to be wellposed in [2]. Below, in the simulation of geometrically and physically nonlinear problems in solid mechanics, we use the variational principles of quasi-static strain [3]. The wellknown principles of the construction of iterative procedures are based on variational inequalities. The formulation of problems in terms of variational inequalities is possible when the functionals involved are convex, which is ensured by taking into account sufficient uniqueness conditions for boundary value problems [4]. This formulation makes it possible to determine the convergence conditions for iterative methods.

\section{FORMULATION OF SHAPING PROBLEMS}

Let $V \subset R^{3}$ be a bounded domain with a sufficiently regular boundary $S$. Denote by $u=\left(u_{1}, u_{2}, u_{3}\right), \tilde{u}=\left(\tilde{u}_{1}, \tilde{u}_{2}, \tilde{u}_{3}\right)$ the vectors of current and residual displacements; $u, \tilde{u} \in\left[W_{2}^{1}(Q)\right]^{3}, Q=V \times\{0 \leq t \leq T\}$.

Consider the quasi-static shaping problem with allowance for small deformations and large displacements and rotations (general Lagrangian formulation), including inelastic strain and elastic unloading. The inelastic strain and elastic unloading problems can be represented in the form of a quasistatic variational principle with the functional:

$$
\begin{gathered}
J(\dot{u}, \dot{\tilde{u}})=\frac{1}{\varepsilon_{1}}\left\|\dot{u}-\dot{u}^{*}\right\|_{S}{ }^{2}+a(u, u)+a(\dot{\tilde{u}}, \dot{\tilde{u}})+\frac{1}{\varepsilon_{2}}\left\|\dot{\tilde{u}}-\dot{\tilde{u}}^{*}\right\|_{S}{ }^{2} \\
\varepsilon_{1}>0, \varepsilon_{1} \rightarrow 0, \varepsilon_{2}>0, \varepsilon_{2} \rightarrow 0,
\end{gathered}
$$

where $\dot{\tilde{u}}_{i}^{*}$ is a given residual displacement rate on $S$; potential form are given by ([3])

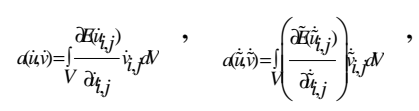

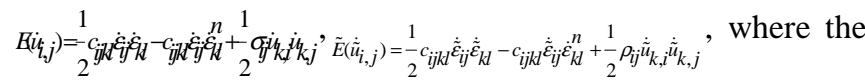
current and residual strain rates are the Green-Lagrange strain tensor rates $\dot{\varepsilon}_{i j}=-\frac{1}{2}\left(\dot{u}_{i, j}+\dot{u}_{j, i}+\dot{u}_{k, i} u_{k, j}+u_{k, i} \dot{u}_{k, j}\right), \dot{\tilde{\varepsilon}}_{i j}=-\frac{1}{2}\left(\dot{\tilde{u}}_{i, j}+\dot{\tilde{u}}_{j, i}+\dot{\tilde{u}}_{k, i} \tilde{u}_{k, j}+\tilde{u}_{k, i} \dot{\tilde{u}}_{k, j}\right)$.

Here, $c_{i j k l}$ are the components of the symmetric elastic constant tensor; $\dot{\varepsilon}_{k l}^{n}$ are the inelastic strain rates (plasticity, creep, plasticity and creep); $i, j, k, l=1,2,3$; dotted letters denote derivatives with respect to time $t$; and double indices with a comma denote differentiation with respect to the coordinate: $u_{i, j}=\frac{\partial u_{i}}{\partial x_{j}}$

In what follows, the symbol $(\cdot, \cdot)_{S}$ denotes the inner product in $L_{2}(S):_{(u, v)_{S}=\int_{S} \sum_{i=1}^{3} u_{i} v_{i} d S}$. The corresponding norm is given by $\|u\|_{S}=\sqrt{(u, u)_{S}}=\left(\int_{S} \sum_{i=1}^{3} u_{i}^{2} d S\right)^{1 / 2}$. 
Alternative constitutive relations written for the current and residual rates of the first Piola-Kirchhoff stress tensor have the form

$$
\dot{\Sigma}_{i j}=\frac{\partial E\left(\dot{u}_{i, j}\right)}{\partial \dot{i}_{i, j}}=\left(\delta_{i k}+u_{i, k}\right) \dot{\sigma}_{k j}+\dot{u}_{i, k} \sigma_{k j}, \dot{\mathrm{P}}_{i j}=\frac{\left.\partial \tilde{E} \dot{u}_{i, j}\right)}{\partial \dot{u}_{i, j}}=\left(\delta_{i k}+\tilde{u}_{i, k}\right) \dot{\rho}_{k j}+\dot{\tilde{u}}_{i, k} \rho_{i j} \cdot
$$

The sufficient conditions are given by $[3,4]$

$$
\int_{V} \Delta \dot{\Sigma}_{i j} \Delta \dot{u}_{i, j} d V>0, \int_{V} \Delta \dot{\mathrm{P}}_{i j} \Delta \dot{\tilde{u}}_{i, j} d V>0
$$

for all pairs of continuously differentiable displacement rate fields taking preset values on the boundary. Here, $\Delta$ denotes the difference between solution-corresponding quantities in any two different forms of strain.

\section{ITERATIVE METHOD FOR SOLVING INVERSE SHAPING PROBLEMS}

Let the sufficient conditions for the uniqueness of a solution of strain problem and unloading problem hold. Then the functional in eqn (1) are not only stationary, but also reach an absolute minimum for a real type of strain (see [4], [5]).

An iterative analogue of the variational inequalities for functional eqn (1) has the form

$$
\begin{aligned}
& A_{1}{ }^{k}\left(\dot{u}^{k+1}-\dot{u}^{k}, v-\dot{u}^{k+1}\right)_{S}+a\left(\dot{u}^{k}, v-\dot{u}^{k+1}\right)+ \\
& +a\left(\dot{\tilde{u}}^{k}, \tilde{v}-\dot{\tilde{u}}^{*}\right)+A_{2}{ }^{k}\left(\dot{\tilde{u}}^{*}-\dot{\tilde{u}}^{k}, \tilde{v}-\dot{\tilde{u}}^{*}\right)_{S} \geq 0, \forall \dot{v}, \forall \dot{\tilde{v}},
\end{aligned}
$$

$$
\text { where } A_{1}{ }^{k}>0, A_{2}{ }^{k}>0, k=1,2, \ldots, \lim _{k \rightarrow \infty} A_{1}{ }^{k}=\infty, \lim _{k \rightarrow \infty} A_{2}{ }^{k}=\infty \text {. }
$$

Then iterative process eqn (2) for solving the inverse shaping problem is represented as [5]

$$
\dot{u}_{i}^{k+1}=\dot{u}_{i}{ }^{k}+\alpha^{k}\left(\dot{\tilde{u}}_{i}{ }^{*}-\dot{\tilde{u}}_{i}^{k}\right), \quad \alpha^{k}=\frac{A_{2}{ }^{k}}{A_{1}^{k}}, 0<\alpha^{k}<2 .
$$

To solve inverse shaping problems for structural elements, such as thin plates, it is sufficient to find a displacement function.

Applying the finite element method $[3,6]$ to the functional of the variational principle for inverse problems with allowance for the unloading displacement $\dot{\mathbf{w}}^{e}=\dot{\mathbf{w}}-\dot{\tilde{\mathbf{w}}}$, we obtain two vector equations

$$
\mathbf{K} \dot{\mathbf{w}}=\dot{\mathbf{F}}, \quad \tilde{\mathbf{K}} \dot{\mathbf{w}}^{e}=\dot{\tilde{\mathbf{F}}}\left(\dot{\boldsymbol{\sigma}}_{0}\right)
$$

where $\dot{\mathbf{w}}, \dot{\tilde{\mathbf{w}}}$ are the velocities of the node parameters describing the inelastic strain displacement $w\left(x_{1}, x_{2}\right)$ and the residual displacement $\tilde{w}\left(x_{1}, x_{2}\right) ; \mathbf{K}, \tilde{\mathbf{K}}$ are the tangent stiffness matrices; and $\dot{\mathbf{F}}$ us the vector of external forces.

The second equation in eqn (4) is an unloading problem with initial stresses and strains obtained by solving the inelastic strain problem.
Consider a square plate of thickness $h$ with a side length $a$. Suppose that a plate displacement is given that models torsion [7] in the form of node displacements in the normal direction to the plate surface.

For a more complete analysis, the volume formulation of the problem is considered. The elastic, plastic and creep characteristics of the v95och material (aluminum alloy) were used in the computations.

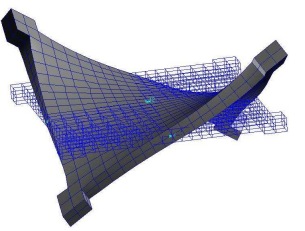

\section{FIGURE I. MODEL OF A PLATE AND ITS ANTICIPATED SHAPE.}

The anticipated shape of the plate ensuring a given curvature after elastic unloading was computed using the iterative method with different constant coefficients $\alpha$. Fig. 1 shows the given residual shape of the plate for which the anticipated shape was found and the flat model of the plate. Fig. 2 demonstrates the convergence of the iterative method eqn (3) with different constant coefficients in the mean-square norm $_{e}=\left(\Sigma_{S}\left(\tilde{\mathbf{w}}^{i}-\tilde{\mathbf{w}}^{0}\right)^{2}\right)^{1 / 2}$, where $S$ is the lower surface of the plate and $i$ is the iteration number.

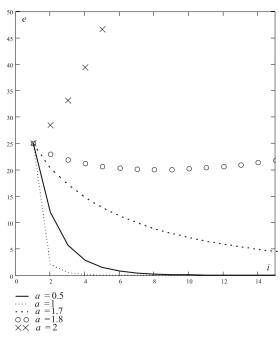

FIGURE II. CONVERGENCE FOR VARIOUS COEFFICIENTS.

An iterative method for solving inverse shaping problems in the general Lagrangian formulation was constructed, and its convergence conditions were determined. Successive approximations were found by applying the finite element method in the MSC. Marc engineering analysis package. The algorithms developed can be used in industrial applications, such as the shaping of aircraft wing panels [8].

This work was supported by the Ministry of Education and Science of the Russian Federation within the state job (project code No. 909).

\section{REFERENCES}

[1] Banshchikova, I. A., Gorev, B. V. and Sukhorukov, I. V. Two dimensional problems of beam forming under conditions of creep. Appl. Mech. Tech. Phys., 43, pp. 448-456, 2002.

[2] Tsvelodub, I. Yu. Certain geometric nonlinear problems of shaping inelastic plates and shallow shells. Prikl. Mekh. Tekh. Fiz., 46 (2), pp. 151-157, 2005.

[3] Korobeinikov, C. H. Nonlinear Deformation of Solids, Sib. Otd. Ross. Akad. Nauk: Novosibirsk, 2000 [in Russian].

[4] Hill, R. On uniqueness and stability in the theory of finite elastic strain. J. Mech. Phys. Solids, 5 (4), pp. 229-241, 1957. 
[5] Bormotin, K. S. Iterative method for solving geometrically nonlinear inverse problems of structural element shaping. Computational mathematics and mathematical physics, 53(12), pp. 1908-1915, 2013.

[6] Zienkiewicz, O. C. The Finite Element Method in Engineering Science, McGraw Hill: New York, 1971; Mir: Moscow, 1975.

[7] Oleinikov, A. I., Korobeinikov, S. N., Gorev, B. V. and Bormotin, K. S. Mathematical modeling of creep in metal products of materials with different tension and compression properties. Vychisl. Metody Program, 9, pp. 346-365, 2008.

[8] Annin, B. D., Oleinikov, A. I. and Bormotin, K. S. Modeling of forming of wing panels of the SSJ 100 aircraft. J. Appl. Mech. Tech. Phys., 51, pp. 579-589, 2010. 